\title{
Diffusion and adoption of the surgical robot in urology
}

\author{
Anup A. Shah, Jathin Bandari, Daniel Pelzman, Benjamin J. Davies, Bruce L. Jacobs \\ Department of Urology, University of Pittsburgh School of Medicine, Pittsburgh, PA, USA \\ Contributions: (I) Conception and design: BL Jacobs, BJ Davies, J Bandari, AA Shah; (II) Administrative support: BL Jacobs, BJ Davies; (III) Provision \\ of study materials or patients: All authors; (IV) Collection and assembly of data: AA Shah, J Bandari, D Pelzman; (V) Data analysis and interpretation: \\ All authors; (VI) Manuscript writing: All authors; (VII) Final approval of manuscript: All authors. \\ Correspondence to: Anup A. Shah, MD. Department of Urology, University of Pittsburgh School of Medicine, $34715^{\text {th }}$ Ave, Suite 701, Pittsburgh, PA \\ 15213, USA. Email: shahaa2@upmc.edu.
}

\begin{abstract}
Over the last two decades, robotic surgery has become a mainstay in hospital systems around the world. Leading this charge has been Intuitive Surgical Inc.'s da Vinci robotic system (Sunnyvale, CA, USA). Through its innovative technology and unique revenue model, Intuitive has installed 4,986 robotic surgical systems worldwide in the last two decades. The rapid rate of adoption and diffusion of the surgical robot has been propelled by many important industry-specific factors. In this review, we propose a model that explains the successful adoption of robotic surgery due to its three core groups: the surgeon, the hospital administrator, and the patient.
\end{abstract}

Keywords: Robotic surgery; robotic prostatectomy; technology adoption; intuitive surgical; da Vinci robot

Submitted Mar 21, 2019. Accepted for publication Nov 15, 2019.

doi: $10.21037 /$ tau.2019.11.33

View this article at: http://dx.doi.org/10.21037/tau.2019.11.33

\section{History of robotic surgery}

Robotic surgery is rooted in the history of laparoscopic surgery. In 1986, the development of the first video computer chip allowing magnification and projection of images onto television screens sparked a worldwide adoption of laparoscopic surgery (1). This rapid rise of laparoscopic procedures led to an interest in exploring laparoscopy's capabilities in the remote setting, ultimately leading to the development of the first robotic surgical systems. One of the first such systems was the Green Stanford Research Institute Telepresence Surgical System (GTSS), a collaboration between multiple investigators beginning in the 1980s, led by Dr. Phillip Green at Stanford Research Institute (2). This system, similar to contemporary "master-slave" robotic surgical systems, included a remote operative site, surgical workstation, and three-dimensional visualization of the surgical site. However similar to standard laparoscopic instruments, the Green Surgical System's instruments maintained only four degrees of freedom (3). In 1992, this endeavor gathered momentum due to the involvement and funding from the United States Defense
Advanced Research Projects Agency (DARPA), which envisioned a robotic tele-surgical system allowing surgeons to perform live remote surgery in the battlefield (4). It was through this initial DARPA-funded Advanced Biomedical Technologies Program that the Automated Endoscopic System for Optimal Positioning (AESOP) was developed by Computer Motion, Inc. in 1993.

After the Green Surgical System team was able to perform the first remote tele-surgical procedure in 1994 on an ex vivo porcine intestine, the concept piqued the interest of Dr. Frederic Moll, a general surgeon who had already founded and sold two companies that developed laparoscopic tools-Origin Medisystems, Inc. and Endotherapeutics $(5,6)$. Moll enlisted the help of electrical engineer Robert Younge and John Freund, a Harvard MBA, and in 1995, they successfully negotiated technology licenses from Stanford Research Institute, International Business Machines Corporation and Massachusetts Institute of Technology to form Intuitive Surgical Devices, Inc. (later changed to Intuitive Surgical, Inc.). By the spring of 1996, they had adapted the GTSS model to incorporate the articulating EndoWrist ${ }^{\mathrm{TM}}$ technology that allowed seven 


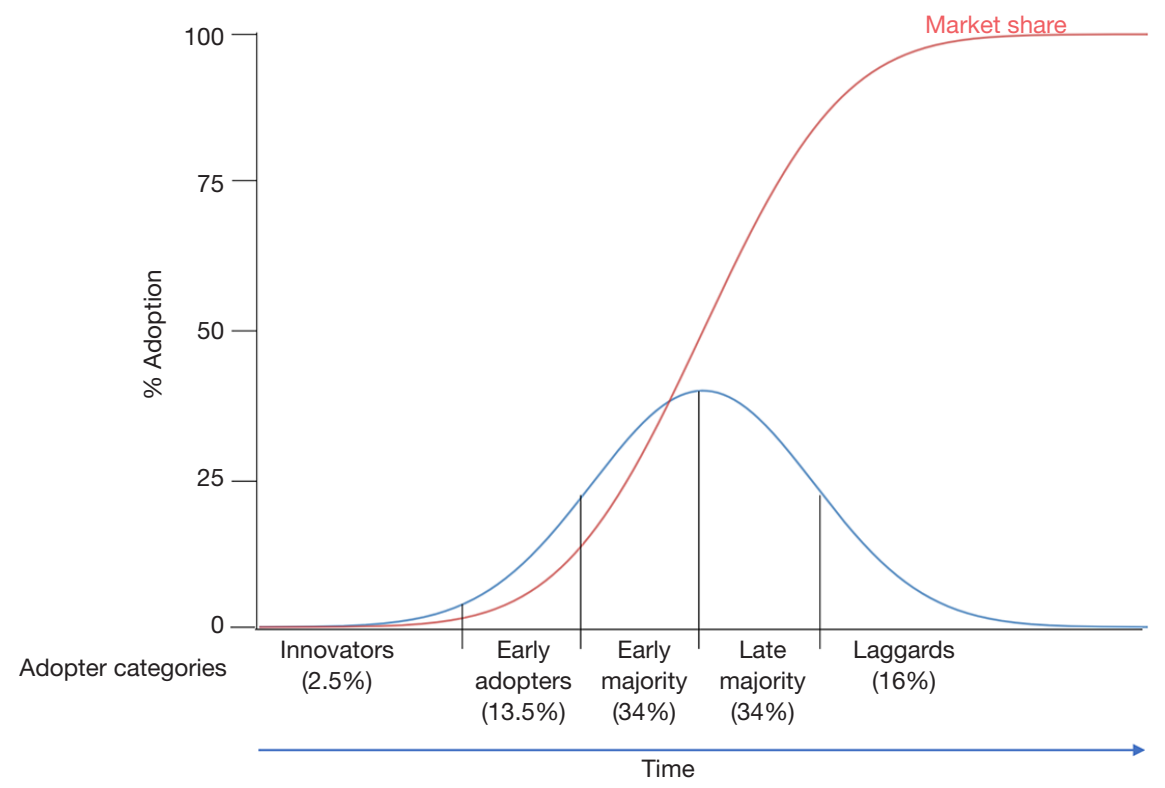

Figure 1 The "diffusion of innovation" model. As consumers adopt a technology (blue curve), its overall market share increases in logistic fashion (red curve) until market saturation is reached. Near the end of the "early majority" phase at roughly $50 \%$ overall adoption, a critical mass is achieved, after which a technology is generally able to sustain itself (20).

degrees of wrist motion to mimic the human wrist, helping Intuitive separate itself from its competitors (3). Rather than focus on telemedicine, Intuitive sought to leverage the EndoWrist ${ }^{\mathrm{TM}}$ technology to facilitate adoption of their technology.

Over the next 3 years, Intuitive would continue to revise and adapt its robot and ultimately registered with the Securities and Exchange Commission in 1998. Notably at that time, the document made no mention of radical prostatectomy as one of the da Vinci's potential uses (7). However, by 2000, multiple groups were reporting use of the da Vinci to perform robotic prostatectomy with the assistance of the EndoWrist ${ }^{\mathrm{TM}}$ technology (8-11). and in May of 2001, the da Vinci surgical system received Food and Drug Administration (FDA) approval for prostate surgery. As of December of 2018, Intuitive Surgical sold 4,986 da Vinci robotic surgical systems worldwide, with 3,196 da Vinci systems installed in the United States (12).

\section{Robotic surgery in urology}

Urologists have been at the forefront of robotics adoption. Since the 2001 FDA approval for da Vinci-assisted prostate surgery, robotic prostatectomy has become the most commonly performed robotic oncologic procedure in the United States (13). By 2003, it was estimated that robotic prostatectomy had an approximately $22 \%$ market share of all radical prostatectomies being performed in the United States (14). The rapid adoption of the robotic prostatectomy placed urology in the center of Intuitive's marketing strategy, which prompted the use of the da Vinci in other genitourinary oncologic procedures-robotic partial nephrectomy in 2002, radical cystectomy in 2003, retroperitoneal lymph node dissection in 2006 and inguinal lymphadenectomy in 2009 (15-19). By 2013, Oberlin estimated that the market share of robotic prostatectomy had increased to $85 \%$, highlighting the rapid rate of diffusion of the surgical robot in urology (14).

Technology adoption can be modeled according to Everett Rogers' pioneering work in his 1962 textbook, Diffusion of Innovations: Innovators, Early Adopters, Early Majority, Late Majority and Laggards (Figure 1). Rogers identified a point of critical mass at about $50 \%$ adoption rate, after which a technology could sustain itself (20). This model was used to measure the adoption of robotic prostatectomy from 2003 to 2010 wherein the Innovator phase was prior to 2005, the Early Adopter phase was between 2005 and 2007, and the Early Majority phase was after 2007 (21). As the phases progressed, the utilization of robotic prostatectomy shifted from a small group of 


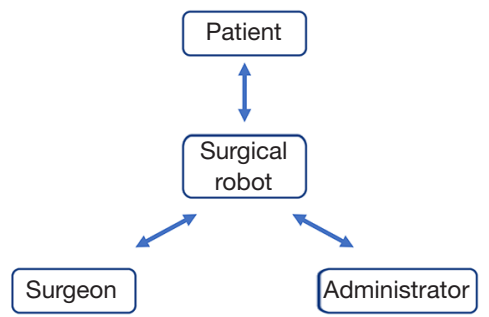

Figure 2 Adoption of the robot requires engagement from three essential stakeholders: patient, surgeon, and hospital administrator.

individual high-volume surgeons to larger hospitals and teaching institutions with larger practices. Additionally, the group noted significant centralization of robotic prostatectomy to high-volume centers.

Utilizing the Diffusion of Innovations model, the time frame for achieving market dominance is variable based on a host of industry-specific factors. In our examination of the surgical robot in urologic oncology, we propose that widespread adoption required engagement from three market participants: the surgeon, the hospital administrator, and the patient (Figure 2).

\section{The surgeon}

The surgeon's involvement is critical to device adoption, because without the approval of the surgeon, procedures cannot be performed even with consent from the patient and hospital administrator arms of our model. The robotic prostatectomy's successes correspond to the failures of surgeons to adopt the laparoscopic radical prostatectomy. Unlike the laparoscopic cholecystectomy which was first performed in the United States in 1988, and had reached an estimated $80 \%$ market saturation 4 years later, the laparoscopic radical prostatectomy was much slower to be adopted (22). Although the first laparoscopic radical prostatectomy was completed in 1992 by Schuessler, by 1997 there had only been a single published case series on laparoscopic radical prostatectomy, published by the same group (23). In that first case series, the mean operative times for the first nine patients was 9.4 hours. Guillonneau later published a 65-patient case series in 1999 with a mean operative time of 4.5 hours, indicating that acceptable operative times could be achieved by some surgeons with significant experience (24).

Despite the $52 \%$ reduction in operative time, the laparoscopic radical prostatectomy struggled to gain traction in the urologic community due to its steep learning curve. The number of cases to achieve competency is estimated to be between 200-750 cases. In comparison, the learning curve for robotic prostatectomy to bring operative times below 4 hours was approximately 40 cases $(25,26)$. By enabling competency with a fewer number of cases, likely due to the da Vinci's EndoWrist ${ }^{\mathrm{TM}}$ technology, the average urologist could adopt the robotic prostatectomy with greater ease than the laparoscopic approach. In addition to the shorter learning curve, the EndoWrist ${ }^{\mathrm{TM}}$ also offers surgeons improved ergonomics. A study comparing musculoskeletal ergonomic parameters of open, laparoscopic and robotic prostatectomy showed that neck and/or back pain was reported in $50 \%$ and $56 \%$ of surgeons after open and laparoscopic approach, respectively, but in only $23 \%$ of surgeons operating robotically (27).

Dwindling competition enabled the da Vinci surgical system to attain widespread adoption. Prior to 2003, the only major competitor Intuitive Surgical faced in this market was the Zeus Robotic Surgical System from Computer Motion Inc. Utilizing the AESOP voice-controlled laparoscopic camera, the Zeus had two additional robotically controlled arms that reproduced the movements that a surgeon made on laparoscopic instruments at the console, but lacked the range of motion of the EndoWrist ${ }^{\mathrm{TM}}$ technology. The Zeus received FDA approval in 2001 for use in abdominal surgery, however the limited range of motion did not translate into natural motions experienced in open surgery. Gill studied the two systems head-to-head in a cohort of pigs, and noted a significantly decreased operative time with the da Vinci compared to the Zeus (28). The comparative ease of use over the Zeus gravitated more urologists to the da Vinci over time, and the Zeus was removed from the market after the merger of Intuitive Surgical and Computer Motion in 2003 (6). After initial adoption was achieved, subsequent innovations required less marketing and broadened the spectrum of possible surgeries. These advancements include the fourth surgical arm, dual console to enable teaching, high definition imaging, upgraded systems with improved ergonomics $(\mathrm{Si}, \mathrm{Xi})$, and now the single-port surgical system (29).

\section{The hospital administrator}

Since capital expenditures average $\$ 1.45$ million dollars per robot, the device manufacturer must be able to justify the cost of the system while also demonstrating its superior or at least equivalent efficacy over the existing standard of care to 
the hospital administrator (12). However, despite the lack of level 1 evidence supporting improved outcomes of robotic prostatectomy over the open approach, specific individual benefits of robotic prostatectomy were heavily touted and allowed the da Vinci to gain significant momentum among hospital administrators (30-32). One of these benefits was the decreased length of stay associated with robotic prostatectomy. In the first published pilot study of robotic prostatectomy by Menon in 2002, 63\% of patients were able to be discharged within 24 hours compared to $0 \%$ in the open cohort (33). This length-of-stay figure was used to justify the high cost of the robot, as the projected cost benefit of decreased length-of-stay offset the initial fixed costs of the device. Since length-of-stay is a variable cost and device installation is a fixed cost, robotic prostatectomy was theorized to be cost-effective at high case volumes. However, cost-effectiveness studies have demonstrated higher costs associated with robotic prostatectomy even at high utilization rates due to significant associated variable costs involving robotic instruments and service contracts (34-38).

Intuitive Surgical utilizes a recurring revenue model, by which a dependent good (the da Vinci robot) is sold at a loss, but is paired with a consumable good (the limited-use surgical instruments) that ultimately generate the profit for the company. Da Vinci robots are installed at a fixed price, ranging from $\$ 500,000$ to $\$ 2.5$ million, while the repeated instrument purchases (range $\$ 700-\$ 3,500$ per procedure) and service contracts (range: $\$ 80,000-\$ 190,000$ per year) generate a majority of Intuitive's revenue (12). Switching costs are substantial for the hospital due to several factors, including: (I) the desire to increase market share by offering a new technology, (II) the misguided assumption that high utilization rates will offset the overall cost of the technology, (III) multi-year service contracts disincentivizing early exit and (IV) trade-in or upgrade programs which subsidize subsequent robot installments (12). In certain circumstances, a robot installment may be sold at a loss with the expectation of recuperating profits in subsequent years. Until 2003, Intuitive Surgical suffered an aggregate net loss of $\$ 81.68 \mathrm{M}$ over 210 installed robots-a loss of $\$ 388,962$ per robot (39). Thus, it is the number of procedures, not the installed base, that determines profits. This is consistent with cost-analysis findings that variable costs are the biggest component of hospital costs $(34,35)$. As new robotic systems enter the market, Intuitive's monopoly on robotic surgery may dwindle. This competition can diminish the cost of robotic procedures.

\section{The patient}

The last group that has played a critical role in the adoption and subsequent diffusion of the surgical robot in urologic oncology is the patient. In his 1992 book "Technopoly: the surrender of culture to technology", Postman describes the concept of technopoly, which refers to a "deification of technology" in which "the culture seeks its authorization in technology, finds its satisfactions in technology, and takes its orders from tecbnology" (40). This concept can be loosely applied to the patient populations that were electing to undergo robotic prostatectomy in the early to mid-2000s, in that the enthusiasm for robotic technology could have contributed to its increasing use across the United States. It can similarly be applied to the physicians that embrace robotics with unbridled enthusiasm. This has been evidenced by the fact that between 2003 and 2010, despite a decrease in the overall radical prostatectomy rate, robotic prostatectomy rates continued to rise and occupied a larger market share of all radical prostatectomies (21).

Intuitive was also adept at capitalizing on direct-toconsumer advertising, or advertising directed to the consumer of the end product-in this case, patientswithout the involvement of an intermediary. This type of advertising has been more heavily regulated by the FDA for pharmaceuticals than medical device manufacturers. For medical device manufacturers, unlike pharmaceuticals, the FDA does not require a randomized controlled trial to prove a device's efficacy and safety, but only that the device be shown to be equivalent to one existing on the current market (41). Additionally, hospital and physician marketing towards patients is not regulated by the FDA, but rather the Federal Trade Commission (FTC), which has historically enforced much looser standards with regards to marketing as they oversee a much broader scope of products (42). A study by Mirkin in 2012 examined the internet promotion of robotic prostatectomy and found that $71 \%$ of promotional websites came from hospitals or private physicians/physician groups and that $42 \%$ of websites did not make any mention of a single risk of robotic prostatectomy (43). Data for many of these websites were provided by Intuitive Surgical and today, the company continues to provide access to online promotional materials (44). A combination of limited regulation on marketing to consumers and a cultural affinity towards new technology has driven patients toward the robotic prostatectomy, allowing its adoption and diffusion rates to rise dramatically. 


\section{Future endeavors}

Given the generalizability of our three-point adoption model, the ongoing adoption and diffusion of further advancements within robotic surgery can be analyzed by their ability to capture the surgeon, the hospital administrator, and the patient markets. For example, one recent development that has gained momentum is the completion of the first single-port robotic prostatectomy through a $2.5 \mathrm{~cm}$ periumbilical incision via Intuitive's da Vinci SP Surgical System (45). This technology would appeal to the technopoly-driven patient and surgeon, however would be significantly limited in its appeal to the hospital administrator as it would require the purchase of a separate da Vinci SP Surgical System, slowing its rate of adoption according to our model.

As data transfer speeds continue to improve, it is clear that DARPA's aspirations of true "remote" telesurgery will be a reality soon. The first telesurgery was completed in 2001, when a remote robot-assisted cholecystectomy was performed on a 68-year-old female in Strasbourg, Germany by surgeons in New York (46). The surgeons utilized the Zeus for this procedure with a delay of 155 milliseconds from console to robot. This type of telesurgery has not yet been reproduced with the da Vinci Surgical System on humans. In January 2019, there were reports of the first telesurgery being performed on pigs in China over a $5 \mathrm{G}$ network with an average 100 millisecond delay over 30 miles (47). This remote technique would appeal to patients who prefer to travel less for their procedures, and surgeons who would be able to operate on patients over a wider geographic area. Local hospital administrators, who would not have to keep a full-time specialized surgeon on their permanent staff could still reap the benefits of being able to offer the operation. This may serve to reduce disparities in rural communities and relieve tensions caused by the pleas for regionalizing highrisk procedures to high-volume centers $(48,49)$. Regardless of the new technology and its purported benefits, the application of our described three-point model is useful in analyzing the market forces that drive the adoption or abandonment of these technologies.

\section{Acknowledgments}

Funding: Bruce Jacobs is supported in part by P30CA047904 from the National Cancer Institute, the Henry L. Hillman Foundation, and the Shadyside Hospital Foundation.

\section{Footnote}

Provenance and Peer Review: This article was commissioned by the Guest Editors (Marc C. Smaldone and Jeffrey J. Tomaszewski) for the series "Controversies in Minimally Invasive Urologic Oncology" published in Translational Andrology and Urology. The article has undergone external peer review.

Conflicts of Interest: All authors have completed the ICMJE uniform disclosure form (available at http://dx.doi. org/10.21037/tau.2019.11.33). The series "Controversies in Minimally Invasive Urologic Oncology” was commissioned by the editorial office without any funding or sponsorship. The authors have no other conflicts of interest to declare.

Ethical Statement: The authors are accountable for all aspects of the work in ensuring that questions related to the accuracy or integrity of any part of the work are appropriately investigated and resolved.

Open Access Statement: This is an Open Access article distributed in accordance with the Creative Commons Attribution-NonCommercial-NoDerivs 4.0 International License (CC BY-NC-ND 4.0), which permits the noncommercial replication and distribution of the article with the strict proviso that no changes or edits are made and the original work is properly cited (including links to both the formal publication through the relevant DOI and the license). See: https://creativecommons.org/licenses/by-nc-nd/4.0/.

\section{References}

1. Spaner SJ, Warnock GL. A brief history of endoscopy, laparoscopy, and laparoscopic surgery. J Laparoendosc Adv Surg Tech A 1997;7:369-73.

2. Green PS. Telepresence: dexterous procedures in a virtual operating field. Surgical Endoscopy 1991;57:192.

3. Schneeberger EW, Michler RE. An overview of the intuitive system: the surgeon's perspective. Operative Techniques in Thoracic and Cardiovascular Surgery 2001;6:170-6.

4. Satava RM. Surgical robotics: the early chronicles: a personal historical perspective. Surg Laparosc Endosc Percutan Tech 2002;12:6-16.

5. Kalan S, Chauhan S, Coelho RF, et al. History of robotic surgery. J Robot Surg 2010;4:141-7.

6. Johnson DD. Intuitive Surgical, Inc. International 
directory of company histories. Farmington Hills: St. James Press, 2016.

7. United States Securities and Exchange Commission. Intuitive Surgical I. Form S-1, 1998.

8. Pasticier G, Rietbergen JB, Guillonneau B, et al. Robotically assisted laparoscopic radical prostatectomy: feasibility study in men. Eur Urol 2001;40:70-4.

9. Binder J, Kramer W. Robotically-assisted laparoscopic radical prostatectomy. BJU Int 2001;87:408-10.

10. Rassweiler J, Frede T, Seemann O, et al. Telesurgical laparoscopic radical prostatectomy. Initial experience. Eur Urol 2001;40:75-83.

11. Abbou CC, Hoznek A, Salomon L, et al. Laparoscopic radical prostatectomy with a remote controlled robot. J Urol 2001;165:1964-6.

12. United States Securities and Exchange Commission. Intuitive Surgical I. Form 10-K, 2019.

13. Fantus RJ, Cohen A, Riedinger CB, et al. Facility-level analysis of robot utilization across disciplines in the National Cancer Database. J Robot Surg 2019;13:293-9.

14. Oberlin DT, Flum AS, Lai JD, et al. The effect of minimally invasive prostatectomy on practice patterns of American urologists. Urol Oncol 2016;34:255.e1-5.

15. Gettman MT, Blute ML, Chow GK, et al. Robotic-assisted laparoscopic partial nephrectomy: technique and initial clinical experience with DaVinci robotic system. Urology 2004;64:914-8.

16. Beecken WD, Wolfram M, Engl T, et al. Robotic-assisted laparoscopic radical cystectomy and intra-abdominal formation of an orthotopic ileal neobladder. Eur Urol 2003;44:337-9.

17. Menon M, Hemal AK, Tewari A, et al. Nerve-sparing robot-assisted radical cystoprostatectomy and urinary diversion. BJU Int 2003;92:232-6.

18. Davol P, Sumfest J, Rukstalis D. Robotic-assisted laparoscopic retroperitoneal lymph node dissection. Urology 2006;67:199.

19. Josephson DY, Jacobsohn KM, Link BA, et al. Roboticassisted endoscopic inguinal lymphadenectomy. Urology 2009;73:167-70; discussion 170-1.

20. Rogers EM. Diffusion of innovations. 5th ed. New York: Free Press, 2003.

21. Chang SL, Kibel AS, Brooks JD, et al. The impact of robotic surgery on the surgical management of prostate cancer in the USA. BJU Int 2015;115:929-36.

22. Escarce JJ, Bloom BS, Hillman AL, et al. Diffusion of laparoscopic cholecystectomy among general surgeons in the United States. Med Care 1995;33:256-71.
23. Schuessler WW, Schulam PG, Clayman RV, et al. Laparoscopic radical prostatectomy: initial short-term experience. Urology 1997;50:854-7.

24. Guillonneau B, Vallancien G. Laparoscopic radical prostatectomy: initial experience and preliminary assessment after 65 operations. Prostate 1999;39:71-5.

25. Abboudi H, Khan MS, Guru KA, et al. Learning curves for urological procedures: a systematic review. BJU Int 2014;114:617-29.

26. Ahlering TE, Skarecky D, Lee D, et al. Successful transfer of open surgical skills to a laparoscopic environment using a robotic interface: initial experience with laparoscopic radical prostatectomy. J Urol 2003;170:1738-41.

27. Bagrodia A, Raman JD. Ergonomics considerations of radical prostatectomy: physician perspective of open, laparoscopic, and robot-assisted techniques. J Endourol 2009;23:627-33.

28. Sung GT, Gill IS. Robotic laparoscopic surgery: a comparison of the DA Vinci and Zeus systems. Urology 2001;58:893-8.

29. Bishoff JT, Kavoussi LR. Atlas of laparoscopic and robotic urologic surgery. Philadelphia: Elsevier, 2017.

30. Hu JC, Gu X, Lipsitz SR, et al. Comparative effectiveness of minimally invasive vs open radical prostatectomy. JAMA 2009;302:1557-64.

31. Ilic D, Evans SM, Allan CA, et al. Laparoscopic and robotic-assisted versus open radical prostatectomy for the treatment of localised prostate cancer. Cochrane Database Syst Rev 2017;9:CD009625.

32. Coughlin GD, Yaxley JW, Chambers SK, et al. Robotassisted laparoscopic prostatectomy versus open radical retropubic prostatectomy: 24-month outcomes from a randomised controlled study. Lancet Oncol 2018;19:1051-60.

33. Menon M, Tewari A, Baize B, et al. Prospective comparison of radical retropubic prostatectomy and robotassisted anatomic prostatectomy: the Vattikuti Urology Institute experience. Urology 2002;60:864-8.

34. Tomaszewski JJ, Matchett JC, Davies BJ, et al. Comparative hospital cost-analysis of open and roboticassisted radical prostatectomy. Urology 2012;80:126-9.

35. Bolenz C, Freedland SJ, Hollenbeck BK, et al. Costs of radical prostatectomy for prostate cancer: a systematic review. Eur Urol 2014;65:316-24.

36. Gandaglia G, Sammon JD, Chang SL, et al. Comparative effectiveness of robot-assisted and open radical prostatectomy in the postdissemination era. J Clin Oncol 2014;32:1419-26. 
37. Close A, Robertson C, Rushton S, et al. Comparative costeffectiveness of robot-assisted and standard laparoscopic prostatectomy as alternatives to open radical prostatectomy for treatment of men with localised prostate cancer: a health technology assessment from the perspective of the UK National Health Service. Eur Urol 2013;64:361-9.

38. Lotan Y, Bolenz C, Gupta A, et al. The effect of the approach to radical prostatectomy on the profitability of hospitals and surgeons. BJU Int 2010;105:1531-5.

39. United States Securities and Exchange Commission. Intuitive Surgical I. Form 10-K, 2003.

40. Postman N. Technopoly: the surrender of culture to technology. 1st ed. New York: Knopf, 1992.

41. United States Food and Drug Administration. Overview of device regulation. 2018. Available online: https://www. fda.gov/medicaldevices/deviceregulationandguidance/ overview/default.htm

42. United States Federal Trade Commission. Health care competition. 2018. Available online: https://www.ftc. gov/news-events/media-resources/mergers-competition/ health-care-competition

43. Mirkin JN, Lowrance WT, Feifer AH, et al. Direct-to- consumer Internet promotion of robotic prostatectomy exhibits varying quality of information. Health Aff (Millwood) 2012;31:760-9.

44. da Vinci Surgery Community (Intuitive Surgical, Inc.) Marketing resources. 2019. Available online: https:// www.davincisurgerycommunity.com/Marketing_ Resources? tab1=PE

45. Kaouk J, Bertolo R, Eltemamy M, et al. Single-port robotassisted radical prostatectomy: first clinical experience using the SP surgical system. Urology 2019;124:309.

46. Marescaux J, Leroy J, Gagner M, et al. Transatlantic robot-assisted telesurgery. Nature 2001;413:379-80.

47. Farooqui A. First $5 \mathrm{G}$ remote surgery completed in China: Ubergizmo. 2019. Available online: https://www. ubergizmo.com/2019/01/first-5g-remote-surgery-china/

48. Birkmeyer JD, Lucas FL, Wennberg DE. Potential benefits of regionalizing major surgery in Medicare patients. Eff Clin Pract 1999;2:277-83.

49. Chhabra KR, Dimick JB. Strategies for improving surgical care: when is regionalization the right choice? JAMA Surg 2016;151:1001-2.
Cite this article as: Shah AA, Bandari J, Pelzman D, Davies BJ, Jacobs BL. Diffusion and adoption of the surgical robot in urology. Transl Androl Urol 2021;10(5):2151-2157. doi: 10.21037/ tau.2019.11.33 